Meta

Journal des traducteurs

Translators' Journal

\title{
Le vocabulaire électrotechnique international de la Commission Électrotechnique Internationale
}

\section{P. Feintuch}

Volume 34, numéro 3, septembre 1989

1. Actes du Colloque Les terminologies spécialisées: Approches quantitative et logico-sémantique et 2 . Actes du Colloque Terminologie et Industries de la langue

URI : https://id.erudit.org/iderudit/002738ar

DOI : https://doi.org/10.7202/002738ar

Aller au sommaire du numéro

Éditeur(s)

Les Presses de l'Université de Montréal

ISSN

0026-0452 (imprimé)

1492-1421 (numérique)

Découvrir la revue

Citer cet article

Feintuch, P. (1989). Le vocabulaire électrotechnique international de la

Commission Électrotechnique Internationale. Meta, 34(3), 539-541.

https://doi.org/10.7202/002738ar 


\section{VOCABULAIRE ÉLECTROTECHNIQUE INTERNATIONAL DE LA COMMISSION ELECTROTECHNIQUE INTERNATIONALE}

P. FEINTUCH
Commission Électrotechnique Internationale

Paris, France

La Commission Électrotechnique Internationale (CEI), qui est un organisme de normalisation dans tous les domaines de l'électricité, a entrepris il y a plus de 60 ans, la confection d'un Vocabulaire Électrotechnique International (VEI), destiné à donner des définitions précises, brèves et correctes des notions concernant l'électricité et d'indiquer les termes par lesquels ces notions doivent être désignées. Ces termes et définitions doivent être acceptés internationalement. Initialement, les définitions étaient données en français et en anglais, mais dans les années 1970, le russe a été rajouté. En outre, les termes sont traduits en allemand, espagnol, italien, néerlandais, polonais et suédois, et très prochainement doivent être également traduits en japonais.

Avant d'expliquer comment est conçu le VEI, il faut expliquer sommairement la méthode générale de travail de la CEI. La CEI comporte 41 Comités Nationaux et 83 Comités d'Études (CE). Chacun des Comités d'Études est chargé de la normalisation dans un domaine particulier. Le Comité d'Études no 1 est chargé de la terminologie. Pour établir une nouvelle norme, le Comité d'Études constitue un groupe de travail formé d'experts internationaux; ce groupe de travail rédige un premier document appelé document secrétariat qui est soumis aux observations des 41 Comités nationaux. Compte tenu de ces observations, le Comité d'Études décide s'il faut revoir fondamentalement le document secrétariat ou s'il est possible de soumettre à la procédure de normalisation, constitutive au fait de ce qui est appelé «la règle des six mois»: le document révisé est soumis aux Comités nationaux qui doivent voter affirmativement ou négativement sur lui, en donnant, en cas de vote négatif, les raisons de leur refus.

Lorsqu'un document est accepté par la majorité des Comités nationaux, un Comité de rédaction composé au moins du secrétaire du Comité d'Études, d'un représentant francophone et d'un représentant anglophone est chargé de rédiger la version définitive qui sera publiée par la CEI.

En ce qui concerne le VEI, les grandes lignes de ces règles générales sont appliquées, mais des détails importants viennent modifier la procédure.

Le groupe de travail chargé d'un chapitre du VEI dépend généralement d'un Comité d'Études spécialisé et non du CEI, terminologie. Le CEI n'a la responsabilité directe du groupe de travail que lorsque le chapitre ne correspond pas au domaine d'activité d'un Comité d'Études spécialisé, ce qui est le cas des notions générales et des chapitres relatifs aux techniques des télécommunications.

Le groupe de travail doit définir le domaine de terminologie étudié, faire la liste des notions à définir, les classer dans un ordre logique, les définir en français et en anglais (le russe est rarement utilisé comme langue de travail), éviter dans la mesure du possible de redéfinir des notions déjà définies dans un chapitre du VEI, et rédiger, au nom de son Comité d'Études le document secrétariat qui sera soumis aux Comités nationaux, et 
éventuellement, aux autres organisations internationales de normalisation (ISO, UIT, CIE, VIC, VIPA, etc.).

Un représentant du secrétariat du CEI assiste normalement à la première séance du groupe de travail et à toute réunion importante, afin de rappeler les directives de confection du VEI.

Il faut insister sur l'importance qu'il y a à travailler au moins en deux langues et à rédiger immédiatement les définitions en anglais et en français, afin de profiter de la présence de plusieurs spécialistes pour clarifier ces définitions et éviter des difficultés de traduction de français en anglais ou d'anglais en français.

Les observations des Comités nationaux sont rassemblés par le groupe de travail, qui convoque une réunion élargie qui devra décider de la suite à donner à ce document secrétariat: remise au point d'un nouveau document secrétariat ou transmission au secrétariat du CEI pour diffusion selon la Règle des Six Mois; c'est donc cette réunion élargie et non le $\mathrm{CE}$ spécialisé qui décide de cette suite à donner.

À cette réunion élargie, sont invités les secrétaire et président du CE spécialisé, les secrétaire et président du CEI, des représentants des autres CE spécialisés éventuellement intéressés, des représentants de chacun des Comités nationaux ayant émis des observations importantes, des représentants des autres organisations concernées, un représentant du Bureau Central de la CEI.

En cas d'accord, le CEI envoie un document, identique au précédent ou bien modifié suivant les observations des Comités nationaux pour diffusion suivant la Règle des six mois.

Suivant la réponse à cette Règle des six mois, le CEI refuse le document, l'accepte, ou l'accepte sous réserve de quelques modifications.

En cas de refus, la procédure précédente est reprise. En cas d'acceptation sous réserves de modifications, ces modifications doivent être soumises aux votes des Comités nationaux qui peuvent les accepter ou les refuser.

En cas d'acceptation, le secrétariat du CEI constitue, avec l'aide du CE spécialisé, un Comité de rédaction, chargé de rédiger, en français et en anglais, le document à publier.

Ce document est envoyé au Comité national de l'URSS pour traduction des termes et définitions en russe, ainsi qu'aux Comités nationaux (prochainement sept) chargé des traductions des termes dans leur langue. classes :

Jusqu'à présent, le VEI comporte environ 60 chapitres divers, répartis en huit

1 Notions générales

2 Matériaux

3 Mesure, réglementation et calculs

4 Matériels électriques

5 Matériels électroniques

6 Production, transport et distribution de l'énergie électrique

7 Techniques des télécommunications

8 Applications particulières

Le secrétariat du CEI a établi à diverses reprises un index récapitulant tous les termes français et anglais, précisant les chapitres où ils se trouvent. En 1982, il a établi le Dictionnaire multilingue de l'électricité où sont rassemblés les différents termes et définitions des chapitres du VEI.

Ce dictionnaire comporte une partie où sont classés par mots clés, eux mêmes classés en ordre alphabétique, les termes français, leur définition en français, leur traduction 
en anglais, russe, allemand, espagnol, italien, néerlandais, polonais et suédois. Une autre partie comprend les termes et définitions anglaises, avec leur traduction dans les huit autres langues. Ces deux parties sont réunies en un seul volume.

Un deuxième volume donne les termes en ordre alphabétique russe, allemand, espagnol, italien, néerlandais, polonais et suédois, avec leur traduction en français en anglais.

Une deuxième édition de ce dictionnaire est en préparation et doit être publiée à la fin de 1989. Elle comprendra principalement en plus de l'édition actuelle, les termes concernant les techniques des télécommunications qui n'étaient pas à jour en 1982. Au total, plus de 12000 termes. 